submitted to Journal of Advanced Physics

\title{
First-principles computed electronic and magnetic properties of zincblende alkaline-earth pnictides
}

\author{
K. Özdoğan田 \\ Department of Physics, Yildiz Technical University, 34210 Istanbul, Turkey \\ I. Galanaki $\Psi^{\dagger}$ \\ Department of Materials Science, School of Natural Sciences, University of Patras, GR-26504 Patra, Greece
}

(Dated: October 25, 2018)

\begin{abstract}
Employing first-principle electronic structure calculations, we study the magnetic and electronic properties of the $\mathrm{XY}(\mathrm{X}=\mathrm{Mg}, \mathrm{Ca}, \mathrm{Sr}$ and $\mathrm{Y}=\mathrm{N}, \mathrm{P}, \mathrm{As}, \mathrm{Sb})$ compounds crystallizing in the zincblende structure. The $\mathrm{Ca}$ and $\mathrm{Sr}$ alkaline-earth metal monopnictides are found to be halfmetallic with a total spin magnetic moment per formula unit of $1.0 \mu_{B}$. In the case of the $\mathrm{Mg}$ alloys the p-d hybridization effect is much weaker and only $\mathrm{MgN}$ is a half-metal. Electron counting of the bands explains the Slater-Pauling behavior exhibited by the total spin magnetic moment. We also study for these alloys the effect of deformation taking into account both the cases of hydrostatic pressure and tetragonalization keeping constant either the in-plane lattice parameters or the unit cell volume. Even large degrees of deformation only marginally affect the electronic and magnetic properties of these alloys. Finally, we show that this stands also for the rocksalt structure. Our results suggest that alkaline-earth metal monopnictides are promising materials for magnetoelectronic applications.
\end{abstract}

Keywords: Electronic Structure Calculations, Half-metals, $d^{0}$-ferromagnets, sp-electron ferromagnets, pnictides

PACS numbers: 75.50.Cc, 75.30.Et, 71.15.Mb

\section{INTRODUCTION}

Half-metals are among the most studied magnetic materials due to their potential application in spintronic devices,$\underline{\underline{1}} \underline{\underline{3}}$ These unusual materials present metallic properties for one spin-direction while they behave like semiconductors for the other spin-direction eventually leading to $100 \%$ spin-polarization at the Fermi level. ${ }^{\underline{4}}$ This peculiar property is exhibited by several transitionmetal alloys but lately half-metallic ferromagnetism has been predicted for several alloys which do not include transition-metal atoms. Such alloys are widely known in literature with various names like $d^{0}$ magnets, $p$ ferromagnets or sp-electron ferromagnets $\frac{\underline{5}}{\underline{\underline{n}}}$ These compounds have the advantage of being energy-efficient for applications since they create weak external magnetic fields and thus lead to minimal energy losses. There are several ways to create sp-electron ferromagnets and an extensive review is given in Ref. 5 .

One of the most promising routes to half-metallic $s p$ electron ferromagnets is the growth of I/II-IV/V nanostructures in metastable lattice structures similar to the case of transition-metal pnictides and chalcogenides in the metastable zincblende structure ${ }^{6}$ Several studies to this research direction have appeared following the pioneering papers published by Geshi et $\mathrm{al}^{\underline{\underline{Z}}}$ and Kusakabe et al ${ }^{\underline{8}}$ who have shown using first-principles calculations that $\mathrm{CaP}$, CaAs and $\mathrm{CaSb}$ alloys present half-metallic ferromagnetism when grown in the zincblende structure. Moreover they have shown that the gap appears due to a p-d hybridization mechanism which is similar as we will discuss in the next paragraph for all half-metallic ferromagnetic I/II-IV/V alloys in all three zincblende, wurtzite and rocksalt metastable structures. The spin magnetic moment in these alloys follows a Slater-Pauling behavior with the total spin magnetic per formula unit in $\mu_{B}$ being 8 minus the number of valence electron in the unit cell: $M_{t}=8-Z_{t}$. The eight stems from the fact that in the majority-spin band we have exactly 4 occupied states (one s and three p states) and the rest of the electrons occupy exclusively the minority-spin states. The same reasoning stands even for Heusler compounds like GeKCa and SnKCa which contain no transition metals and have been predicted to exhibit half-metallic ferromagnetism.$\underline{9}$ Evidence of the growth of such nanosctructures has been provided by Liu et al who have reported successful self-assembly growth of ultrathin CaN in the rocksalt structure on top of $\mathrm{Cu}(001) ! \underline{10}$

\section{OVERVIEW OF RECENT LITERATURE}

Following the Refs. 7 and 8 mentioned in the previous paragraph a lot of theoretical studies on sp-electron ferromagnets have appeared based on first principles calculations and we will give a short overview of them. A general trend is that the rocksalt (RS) structure is more stable than the zincblende (ZB) or wurtzite (WZ) structures, in all cases the Slater-Pauling (SP) rule for the total spin magnetic moment is obeyed and the spin magnetic moment is almost entirely concentrated to the anionic sites and the interstitial region while the cations show a spin 
moment one or two orders of magnitude smaller (note that the occupied states are almost exclusively of anionic s- and p-character). Also in most studied cases half-metallicity is conserved for large variations of the lattice constant assuming hydrostatic pressure.

We will start our overview from the alkali-metal based alloys. In 2006 Sieberer and collaborators predicted halfmetallicity for LiN and $\mathrm{Na}(\mathrm{K}) \mathrm{P}(\mathrm{As})$ in the zincblende structure with a total spin magnetic moment of $2 \mu_{B}$ per formula unit (f.u.) 11 and in 2008 Zhang confirmed the half-metallicity also for the alkali-metal carbides $(\mathrm{Li}, \mathrm{K}, \mathrm{Na}) \mathrm{C}$ with a total spin moment of $3 \mu_{B}$ in accordance to the SP rule ${ }^{12}$ After, Zberecki and collaborators have demonstrated that the rocksalt is the most stable structure for alkali-metal nitrides with respect to the zincblende and wurtzite structures 13 These results were independently confirmed by Yan, $\stackrel{14}{\underline{1}}$ and by Gao and collaborators who have also shown that among the alkali metal phosphides and arsenides, only KP and KAs are half-metallic ferromagnets $\underline{15}$ Finally, Gao et al have also studied the alkali-metal sulfides $(\mathrm{Li}, \mathrm{Na}, \mathrm{K}) \mathrm{S}$ which exhibited a total spin magnetic moment of $1 \mu_{B}$ as expected by the SP rule for both the ZB and the energetically-favored RS structures 16

Gao and collaborators have studied a series of alkalineearth metal compounds with the IVth column elements and found that all $\mathrm{CaSi}, \mathrm{CaGe}, \mathrm{CaC}, \mathrm{SrC}$ and $\mathrm{BaC}$ in

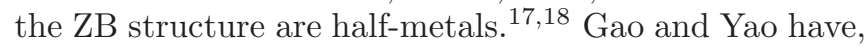
then, studied $\mathrm{SrC}$ and $\mathrm{BaC}$ in more detail and have shown that the RS structure in the most stable against the other metastable structures and found that both RS-SrC and $\mathrm{RS}-\mathrm{BaC}$ are half-metals with the Curie temperature of SrC exceeding the room temperature $(485 \mathrm{~K}) \stackrel{19}{ }$ Dong and Zhao have shown that the alkaline-earth metal carbides retain their half-metallicity up to a hydrostatic pressure of $32 \mathrm{GPa}$ (the volume is reduced by $30 \%$ ) 20 Zhang et al have studied ScC in the WZ structure $\stackrel{21}{\rightleftharpoons}$ and finally Gao have shown that the (110) surface of ZB-CaC keep their half-metallic character contrary to the (001) surfaces. ${ }^{22}$ Also Verma and collaborators have studied the alkaline-earth metal silicides and found that only the ZB-structure is half-metallic contrary to the more-stable RS ferromagnetic structure. ${ }^{23}$

Most of the attention has been focused on the alkalineearth metal (IInd column) compounds with the Vthcolumn elements. As we have already mentioned halfmetallicity has been predicted for the CaP, CaAs and CaSb ZB-alloys $\stackrel{7,8}{7}$ Sieberer et al studied all possible II$\mathrm{V}$ combinations in the $\mathrm{ZB}$ and $\mathrm{WZ}$ structures and found that all alloys containing $\mathrm{Ca}$, Sr and $\mathrm{Ba}$ are half-metallic while only $\mathrm{MgN}$ was half-metallic between the Mg-based compounds $\frac{11}{1 t}$ was also shown in Ref. 11 that the ferromagnetic state is energetically preferable to both the nonmagnetic and the antiferromagnetic configurations. Yao et al have shown that the ZB-alloys containing Bi from

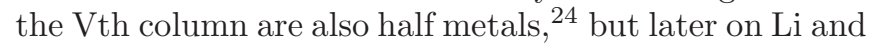
Yu demonstrated that the inclusion of spin-orbit coupling destroys half-metallicity in these Bi-alloys. ${ }^{25}$ Volnianska

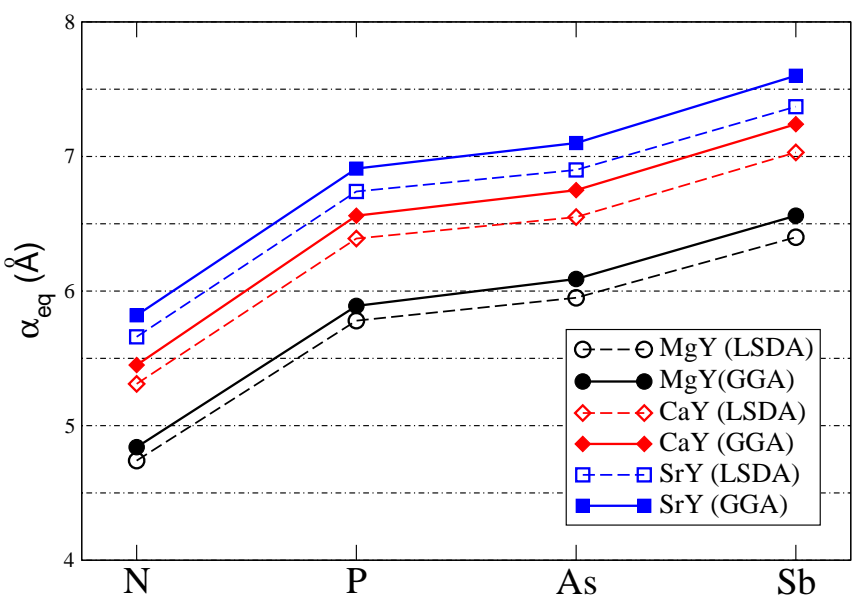

FIG. 1: (Color online) Calculated equilibrium lattice constant in Ångstrøm of several alkaline-earth pnictides assuming the zincblende structure. Total energy calculations have been performed using both the local-spin density (LSDA) and generalized gradient (GGA) approximations to the exchangecorrelation potential in conjunction to the full-potential nonorthogonal local-orbital minimum-basis band structure method (FPLO).

and Boguslawski, as well as Geshi and collaborators have studied the alkaline-earth metal nitrides and have shown that the RS is the most stable structure with formation energies of about $-11 \mathrm{eV}$ per unit cell. ${ }^{26,27}$ Gao et al have shown that among the $\mathrm{RS}$ alloys containing $\mathrm{Ca}$, $\mathrm{Sr}$ or $\mathrm{Ba}$ as a cation and $\mathrm{N}, \mathrm{P}$ or $\mathrm{As}$ as an anion only the nitrides are stable half-metallic ferromagnets with a total spin magnetic moment of $1 \mu_{B}$ and cohesive energies about -9 $\mathrm{eV}$ per formula unit $\stackrel{28}{ }$ Droghetti and collaborators have shown that RS-MgN is in verge of the half-metallicity and suggested that $\mathrm{MgN}$ inclusion upon the $\mathrm{N}$-doping of $\mathrm{MgO}$ should lead to a material suitable for magnetic tunnel junctions $\stackrel{29}{2}$ The most recent studies on nitrides concern the Curie temperature which was found to be well above the room temperature for $\mathrm{CaN}$ and $\mathrm{SrN}$ in both the ZB and RS structures, $\stackrel{30,31}{ }$ and the RS-CaN/ZB-InN and RS-SrN/ZB-GaP (111) interfaces which were found to retain half-metallicity only when the interface is made up from $\mathrm{Ca}-\mathrm{N}$ or $\mathrm{N}-\mathrm{In}$ atoms in the first case and $\mathrm{N}-\mathrm{Ga}$ in the second case 32

\section{MOTIVATION AND COMPUTATION METHOD}

From the discussion in Section III we can conclude that alkaline-earth metal pnictides are very promising for spintronic applications since (i) have a small spin magnetic moment per formula unit $\left(1 \mu_{B}\right)$ and thus create small external magnetic fields, (ii) they present very stable half-metallicity upon hydrostatic pressure, (iii) their equilibrium lattice constant are close to a lot of semiconductors, (iv) results on the $\mathrm{ZB}$ and $\mathrm{RS}$ structures 
TABLE I: Equilibrium lattice constants in Ångstrøm determined through total energy calculations employing the FPLO method within both the LSDA and GGA approximations for all zincblende XY compounds under study. With $m^{\mathrm{X}}$ and $m^{\mathrm{Y}}$ we present the atom-resolved spin magnetic moment in $\mu_{\mathrm{B}}$ at both the cation $(\mathrm{X})$ and anion $(\mathrm{Y})$ sites at the corresponding equilibrium lattice constants, as well as the total spin magnetic moment $\left(m^{\text {total }}\right)$ per formula unit. The Mg-based alloys, with the exception of $\mathrm{MgN}$, have converged to non-magnetic ground states.

\begin{tabular}{|c|c|c|c|c|c|c|c|c|}
\hline \multirow{2}{*}{\begin{tabular}{c|}
$\mathrm{XY}$ \\
compounds
\end{tabular}} & \multicolumn{2}{|c|}{$\alpha_{\mathrm{eq}}(\AA)$} & \multicolumn{2}{|c|}{$m^{\mathrm{X}}\left(\mu_{\mathrm{B}}\right)$} & \multicolumn{2}{|c|}{$m^{\mathrm{Y}}\left(\mu_{\mathrm{B}}\right)$} & \multicolumn{2}{|c|}{$m^{\text {total }}\left(\mu_{\mathrm{B}}\right)$} \\
\hline & LSDA & GGA & LSDA & GGA & LSDA & GGA & LSDA & GGA \\
\hline $\mathrm{MgN}$ & 4.74 & 4.84 & -0.054 & -0.080 & 1.054 & 1.080 & 1.0 & 1.0 \\
\hline $\mathrm{MgP}$ & 5.78 & 5.89 & \multirow{3}{*}{\multicolumn{2}{|c|}{$\begin{array}{l}\text { Non-magnetic } \\
\text { Non-magnetic } \\
\text { Non-magnetic }\end{array}$}} & \multirow{3}{*}{\multicolumn{2}{|c|}{$\begin{array}{l}\text { Non-magnetic } \\
\text { Non-magnetic } \\
\text { Non-magnetic }\end{array}$}} & \multirow{3}{*}{\multicolumn{2}{|c|}{$\begin{array}{l}\text { Non-magnetic } \\
\text { Non-magnetic } \\
\text { Non-magnetic }\end{array}$}} \\
\hline $\mathrm{MgAs}$ & 5.95 & 6.09 & & & & & & \\
\hline $\mathrm{MgSb}$ & 6.40 & 6.56 & & & & & & \\
\hline $\mathrm{CaN}$ & 5.31 & 5.45 & -0.053 & \begin{tabular}{l|l}
-0.073 \\
\end{tabular} & 1.053 & 1.073 & 1.0 & 1.0 \\
\hline $\mathrm{CaP}$ & 6.39 & 6.56 & 0.059 & 0.025 & 0.941 & 0.975 & 1.0 & 1.0 \\
\hline $\mathrm{CaAs}$ & 6.55 & 6.75 & 0.082 & 0.044 & 0.918 & 0.956 & 1.0 & 1.0 \\
\hline $\mathrm{CaSb}$ & 7.03 & 7.24 & 0.144 & 0.102 & 0.856 & 0.898 & 1.0 & 1.0 \\
\hline $\mathrm{SrN}$ & 5.66 & 5.82 & -0.071 & $\begin{array}{l}-0.093 \\
\end{array}$ & 1.071 & 1.093 & 1.0 & 1.0 \\
\hline $\mathrm{SrP}$ & 6.74 & 6.91 & 0.003 & -0.031 & 0.997 & 1.031 & 1.0 & 1.0 \\
\hline SrAs & 6.90 & 7.10 & 0.018 & -0.026 & 0.982 & 1.026 & 1.0 & 1.0 \\
\hline $\mathrm{SrSb}$ & 7.37 & 7.60 & 0.065 & 0.018 & 0.935 & 0.982 & 1.0 & 1.0 \\
\hline
\end{tabular}

suggest high values of the Curie temperature, (v) the half-metallic gaps are wide, and (vi) interfaces with semiconductors retain half-metallicity. In this article we will study in detail (Section IV]) the electronic and magnetic properties of several pinctides in the zinc-blende structure having the chemical formula XY, where the IInd column element $\mathrm{X}$ is $\mathrm{Mg}, \mathrm{Ca}$ or $\mathrm{Sr}$ and the Vth column element Y is N, P, As or Sb. This study is followed (Section $\mathrm{V}$ by an extensive investigation of the properties of these alloys under hydrostatic pressure, tetragonalization keeping the in-plane lattice parameters constant and tetragonalization keeping the volume of the unit cell constant. These latter calculations simulate the effect of growth on various substrates where one of the three cases of deformation can occur. To make our study more complete we have also conducted calculations for RS CaN and $\mathrm{SrN}$ under such deformations.

To perform our calculations we employ the fullpotential nonorthogonal local-orbital minimum-basis band structure scheme (FPLO $)^{33}$ using a dense $20 \times 20 \times 20 \mathrm{k}$-mesh in the Brillouin zone in the reciprocal space to perform integrations. Since we are interested in deformations, we have first to determine the equilibrium lattice constant using total energy calculations for all compounds under study in the ZB lattice structure. In Fig. [1we summarize our results using both the local-spin density (LSDA) $\stackrel{34}{=}$ and generalized-gradient $(\mathrm{GGA})^{35}$ approximations to the exchange correlationpotential. GGA is know to soften the bonds with respect to LSDA and to produce equilibrium lattice constants more close to experimental data (in fact GGA overestimates lattice constants by less than $1 \%$ while LSDA underestimates them by more than 1\%). Both LSDA and GGA curves exhibit exactly the same behavior in Fig. 1 with the LSDA lying under the GGA values as expected. As we move from $\mathrm{Mg}$ to $\mathrm{Ca}$ and then to $\mathrm{Sr}$ for the same $\mathrm{Y}$ element the calculated equilibrium lattice constant be- comes larger since we move from a lighter to a heavier atom. The same trend occurs for one X-element as we move from $\mathrm{N}$ to $\mathrm{P}$ and then to As and $\mathrm{Sb}$. Interestingly the phosphides and arsenides have very close lying equilibrium lattice parameters. As stated above the GGA is expected to give a better estimation of the elastic properties with respect to LSDA while both yield similar description of the magnetic properties for the same lattice constant. In Table \we have gathered our results for all alloys under study using both LSDA and GGA. Our calculated GGA equilibrium lattice constants for $\mathrm{CaP}, \mathrm{CaAs}$ and $\mathrm{CaSb}$ are in excellent agreement with the GGA results by Geshi and collaborators using the full-potential linearized augmented plane waves methods (FLAPW) $\underline{\underline{?}}$ Notice that for MgP, MgAs and MgSb both approximations converged to a non-magnetic solution. For all other alloys both LSDA and GGA give a total spin magnetic moment of $1.0 \mu_{B}$ at the LSDA and GGA equilibrium lattice parameters and thus these alloys are expected to present half-metallic behavior. The atom-resolved spin magnetic moments show a small variation in the second decimal digit between GGA and LSDA. Its origin is the different equilibrium lattice constant produced by LSDA and GGA, and for the same lattice constant both LSDA and GGA produce almost identical spin magnetic moments. Thus in the rest of the paper all presented results have been obtained using the GGA approximation at the GGA equilibrium lattice constants. In general our results agree well with the previous ab-initio studies on these materials discussed in Section 1

\section{EQUILIBRIUM PROPERTIES}

As shown in Table \MgP, MgAs and MgSb converge to non-magnetic solutions while all other alloys seem to be half-metallic ferromagnets as derived from the Slater- 


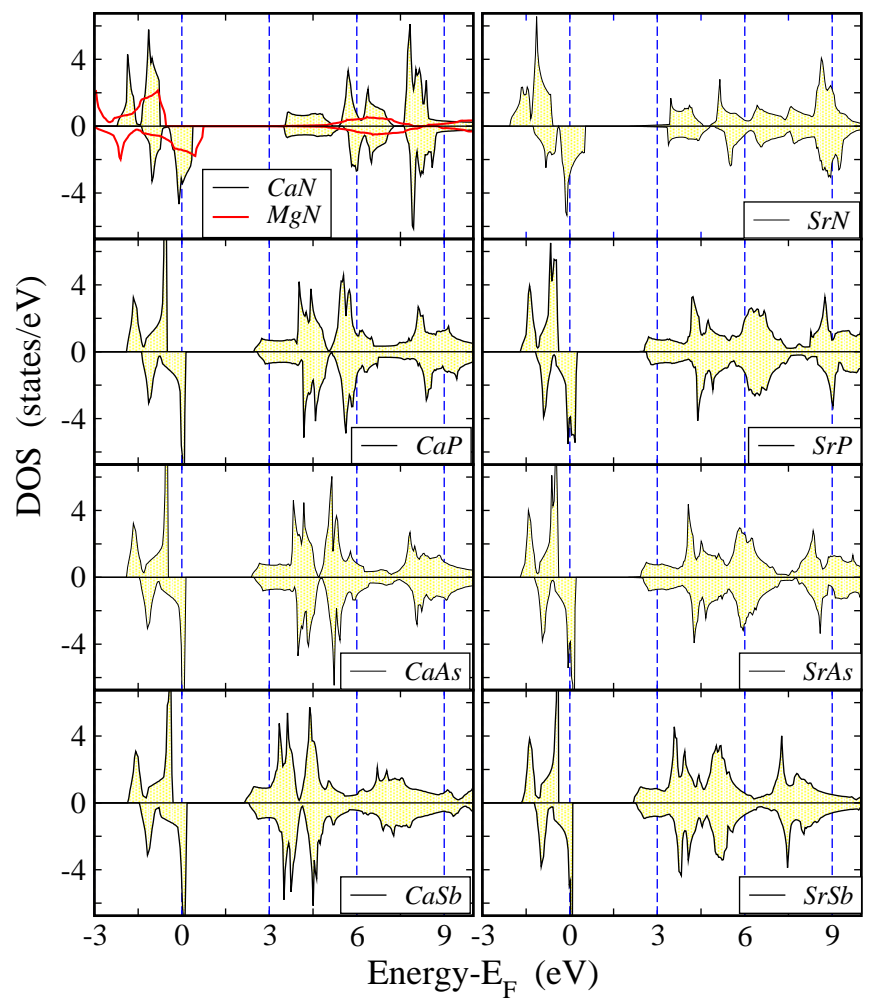

FIG. 2: (Color online) Total DOS in the primitive cell for all studied compounds for a wide energy spectrum within the GGA approximation at the equilibrium lattice constant. The zero energy has been chosen to represent the Fermi energy $\left(\mathrm{E}_{\mathrm{F}}\right)$. The positive DOS values correspond to the majorityspin (spin-up) electrons and the negative DOS values to the minority-spin (spin-down) electrons. The antibonding states lying above both the majority-spin and minority-spin gaps are mainly of cationic d-character while the states below the gaps are mainly of anionic-p character as shown in Fig. 5 Note that we do present the anionic s-states located at about $-10 \sim-12 \mathrm{eV}$ for both spin directions.

Pauling rule discussed in Section 1 which will be again referred to later in the text. In Fig. 2 we have drawn the total density of states (DOS) for all magnetic alloys as a function of the energy setting the Fermi level as the zero energy. Moreover positive DOS values refer to the majority-spin or spin-up electrons and negative DOS values to the minority-spin or spin-down electrons. In all cases we do not represent the s-valence states which are located at about 10 12 eV below the Fermi level. Note that for all compounds under study there are very large gaps in both majority- and minority-spin electronics bands structures. The Fermi level crosses the minorityspin states while it falls within the gap for the majorityspin states and thus we conclude that these alloys are half-metallic ferromagnets where the majority-spin band shows semiconducting behavior while the minority-spin band shows metallic behavior. As we move from $\mathrm{MgN}$ to $\mathrm{CaN}$ and then to $\mathrm{SrN}$ the bands become more narrow while as we move along the Vth column $(\mathrm{N} \rightarrow \mathrm{P} \rightarrow \mathrm{As} \rightarrow \mathrm{Sb})$

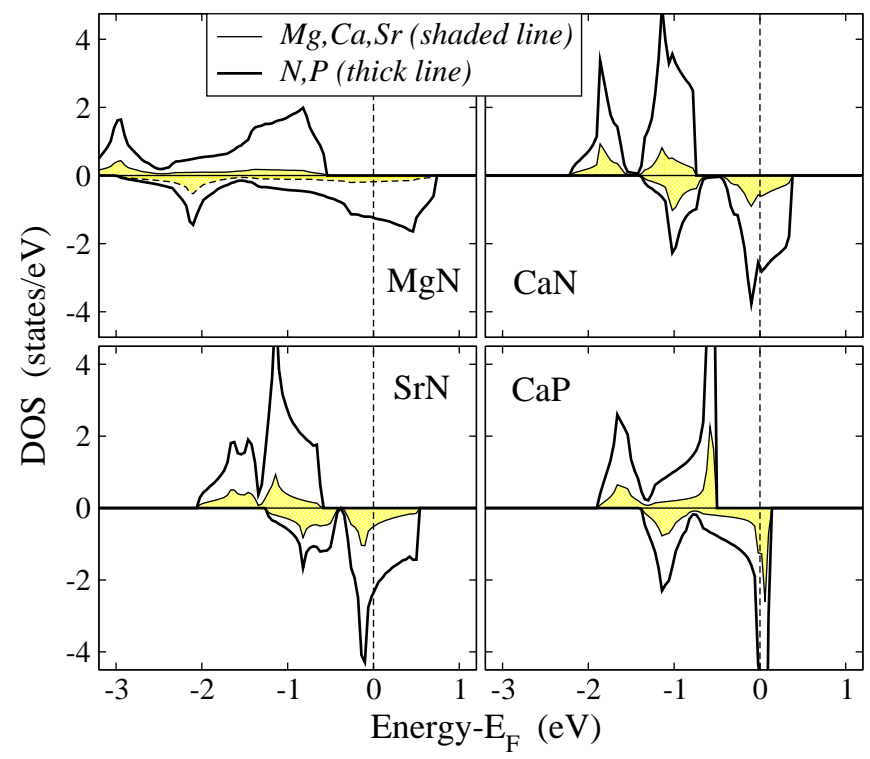

FIG. 3: (Color online) Atom- and spin-resolved density of states (DOS) of the four alloys presented also in Fig. 4 We focus around the Fermi level and do not present the deeplying s-states (see caption of Fig. 2) and the high-energy antibonding states (see Fig. 2 for a broader energy spectrum). Details as in Fig. 2

the gap becomes smaller.

The main weight of the states lie above the Fermi level. This is easy to understand if we examine the origin of the gap discussed in Refs. 7 and 8 . The X-elements from the IInd column $(\mathrm{Mg}, \mathrm{Ca}, \mathrm{Sr})$ provide two valence electrons (occupying e.g. in $\mathrm{Ca}$ the $4 \mathrm{~s}$ states in the free atom) while the anions (N,P,As,Sb) provide 5 valence electrons (e.g. in free atom of As the atomic configuration is $\left.4 \mathrm{~s}^{2} 4 \mathrm{p}^{3}\right)$. In total there are 7 valence electrons per unit cell. The first two occupy the s-valence states created by the anions which lie deep in energy as mentioned above. The p-states of anions (N,P,As,Sb) hybridize strongly with the unoccupied triple-degenerated $\mathrm{t}_{2 g}$ d-states of the cation, which transform following the same symmetry operations in case of lattice with tetrahedral symmetry like the zincblende one, and form bonding and antibonding hybrids which are separated by large energy gaps. The bonding hybrids contain mostly padmixture while the antibonding hybrids are mainly of $\mathrm{d}$ character. The remaining 5 valence electrons occupy the bonding hybrids which are mainly of anionic p-character in such a way that all three majority-spin p-states are occupied while in the minority-spin band the Fermi level crosses the bands so that only the two out of three pstates are occupied. This gives in total a spin magnetic moment per formula unit of exactly $1 \mu_{B}$. In the case of $\mathrm{Ca}$ and $\mathrm{Sr}$ the unoccupied $3 \mathrm{~d}$ and $4 \mathrm{~d}$ states, respectively, are relatively close in energy to the valence p-states of the anion and thus hybridization is strong enough so that all resulting compounds are magnetic. The case of $\mathrm{Mg}$ should be discussed separately since for light $\mathrm{Mg}$ the 
MgN-GGA

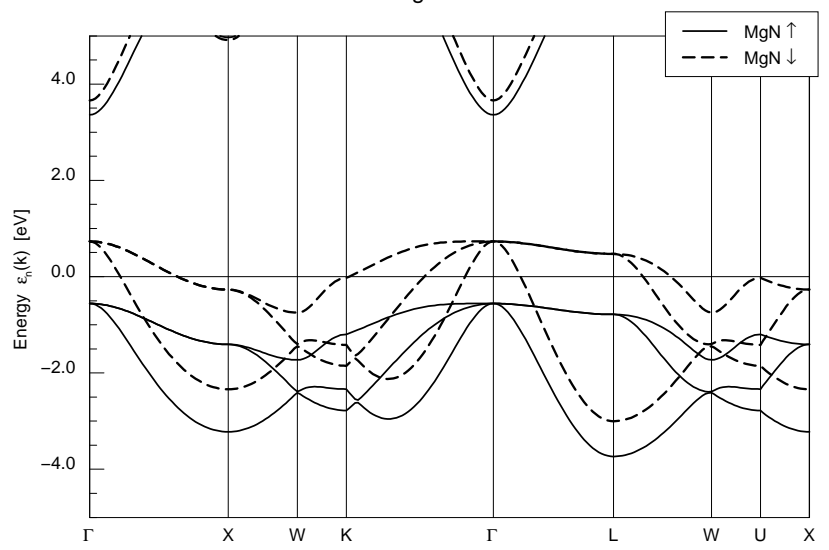

SrN-GGA

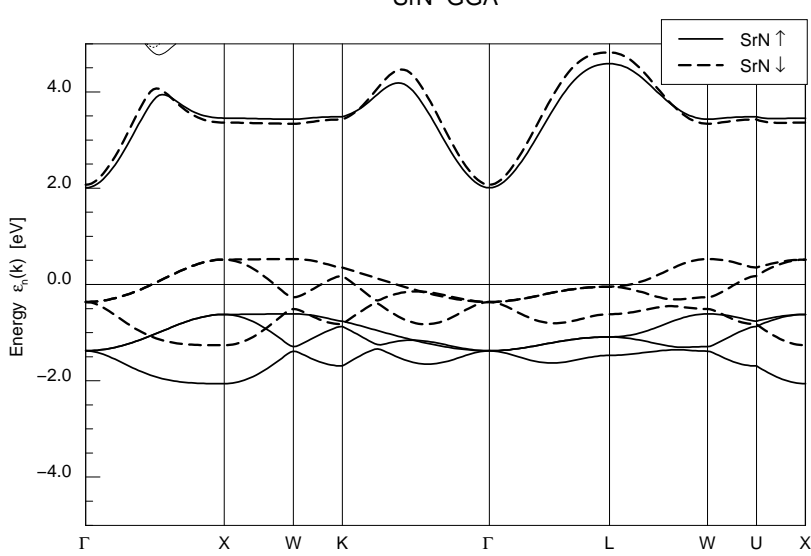

CaN-GGA

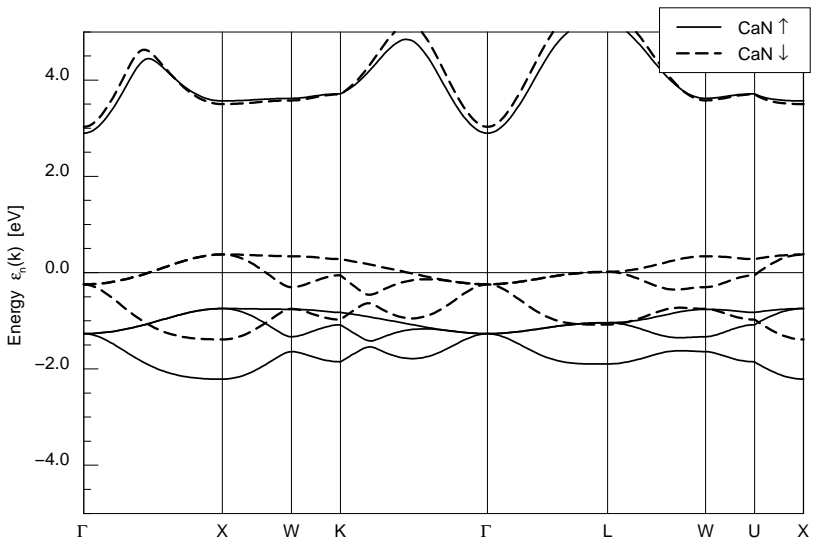

5

FIG. 4: Spin-resolved band structure of $\mathrm{MgN}, \mathrm{CaN}, \mathrm{SrN}$ and $\mathrm{CaP}$ compounds within the GGA approximation along the highsymmetry lines in the Brillouin zone. The zero energy has been chosen to represent the Fermi energy. The solid lines correspond to the majority-spin (spin-up) electrons and the dashed lines to the minority-spin (spin-down) electrons. The deep-lying s-band is not shown; it is located at $10 \sim 12 \mathrm{eV}$ below the Fermi level.

unoccupied 3d states are very high in energy with respect to the occupied states compared to $\mathrm{Ca}$ for which also the first unoccupied d-shell is the $3 \mathrm{~d}$ one. As a result in $\mathrm{MgY}$ alloys the $\mathrm{p}$ - $\mathrm{d}$ hybridization is very weak and the resulting bands are very broad. In $\mathrm{MgP}, \mathrm{MgAs}$ and MgSb the DOS at the Fermi level is weak and these alloys prefer to stay non-magnetic and only $\mathrm{MgN}$ gains energy when it becomes magnetic. Sieberer et al using the FLAPW method within GGA have predicted that $\mathrm{MgP}$ and $\mathrm{MgAs}$ are magnetic with very small magnetic moments ( 0.08 and $0.02 \mu_{B}$ respectively) instead of non magnetic. ${ }^{11}$ The difference should be attributed to the fact that, as shown also on Ref. 11, these compounds are at the verge of being magnetic and a small shift of the predicted Fermi level or a slightly different shape of the predicted DOS by different ab-initio methods could eventually lead to the loss of the magnetism,. The latter compounds can be also seen as a liming case of the Stoner criterion for transition-metal alloys which states that magnetism is the ground state only when the DOS at the Fermi level in the non-magnetic case is quite high and the system gains energy when being magnetic.

The DOS above the gaps in Fig. 2 comes mainly from the unoccupied d-states and exhibits vanishing spin-

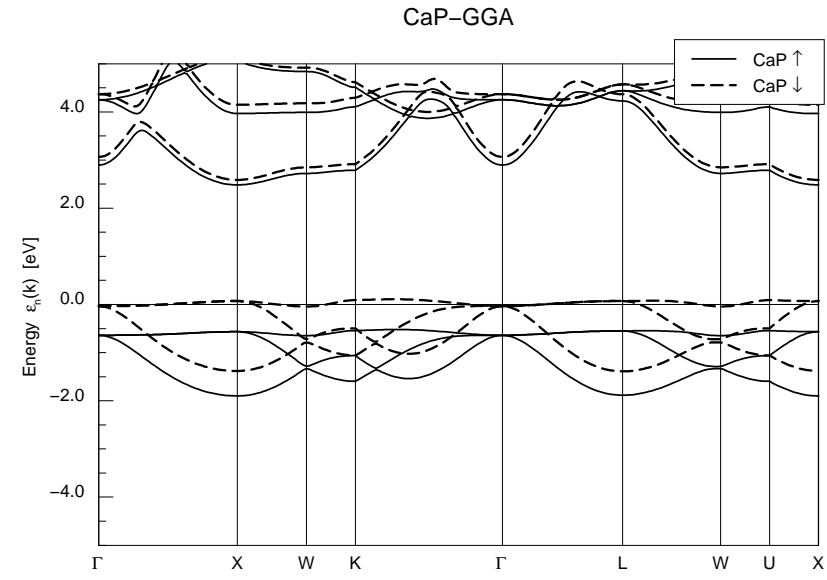


anionic p-DOS. Among the nitrides, $\mathrm{CaN}$ and $\mathrm{SrN}$ exhibit similar DOS curves but MgN differs considerably since the hybridization effect for this alloy is much less intense than for $\mathrm{SrN}$ and $\mathrm{CaN}$. Moreover as we substitute $\mathrm{P}$ for $\mathrm{N}$ in $\mathrm{CaN}$ the bands become more narrow and the exchange-splitting between the majority- and minorityspin bands decreases (difference in the energy position of the mass centers of the bands). All these features can be identified when we look at the band structure for these four alloys and in Fig. 4 we have drawn the bandstructure for both spin-directions in the energy window from -5 to $+5 \mathrm{eV}$ along the high-symmetry axis in the Brillouin zone. Majority-spin (solid lines) and minorityspin (dashed lines) bands have exactly the same shape for all four compounds and they are simply shifted in energy. Similarities are clear if we compare $\mathrm{CaN}$ and $\mathrm{SrN}$ where the difference in the energy positions of the bands is small. In $\mathrm{MgN}$ the bands are much broader while in $\mathrm{CaP}$ the exchange splitting is smaller and with respect to CaN more unoccupied bands appear in the same energy window. As a result of the reduced exchange splitting also the bonding $\mathrm{p}-\mathrm{d}$ hybrids are more narrow. Our calculated band structures for the Ca-based alloys are in good agreement with the ones calculated by $\mathrm{Li}$ and $\mathrm{Yu}$ using a GGA pseudopotential technique $\stackrel{25}{=}$ Finally we should note that our band structure confirms that the states below the gaps are p-d hybrids with mainly p-character, since they are triple degenerated at the $\Gamma$ point, a characteristic of either the $\mathrm{p}$ or the $\mathrm{t}_{2 g}$ states in the lattices of tetrahedral symmetry likes the zincblende one.

In the last paragraph we shall also discuss the spin magnetic moments presented in Table I The cations carry very small spin magnetic moments of less than $|0.1| \mu_{B}$ and almost all spin moment is carried from the anions. The spin magnetic moment reflects directly the imbalance in the total number of occupied states. In Fig. 5 we have drawn both the DOS and the integrated DOS for the bonding p-d hybrids in the case of CaN. We do not include in the integrated DOS the deep-lying s-states which accommodate one electron per spin direction and thus carry no net spin moment. If we integrate the total DOS we have three states per spin direction as expected by the band-structure discussed just above. All the majority-spin states are occupied while the integrated DOS line cross the Fermi level at the value of two. Thus in the minority-spin band only the two out of three states are occupied and the total spin magnetic moment is $1 \mu_{B}$ since we have an imbalance in the electronic charge counting of one. This picture agrees with the Slater-Pauling behavior for the sp-electron ferromagnets which we have discussed in Section 【 If we now look at the Ca resolved DOS in total we have slightly less than 0.5 electrons in the majority-spin band and about 0.65 in the minority-spin band. All majority states are occupied while in the minority-spin band only the 0.5 out of 0.65 are occupied. This small imbalance in the electron counting leads to a small negative spin magnetic moment at the Ca site of $-0.073 \mu_{B}$. Small variation of

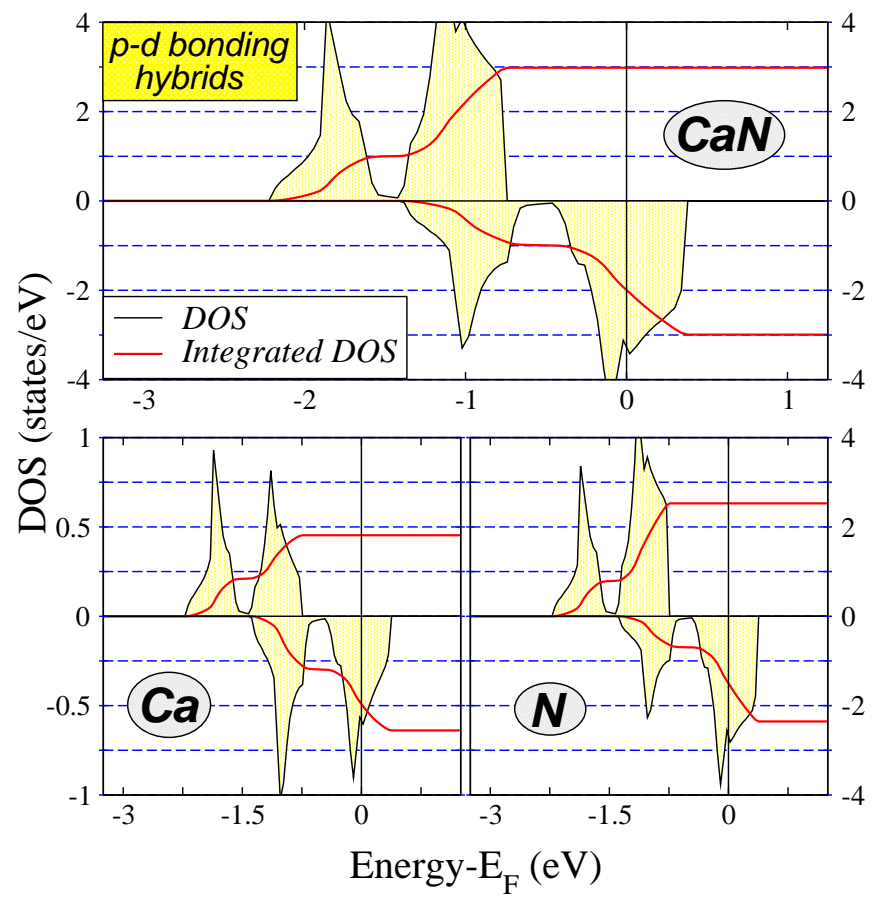

FIG. 5: (Color online) Spin-resolved DOS of the p-d bonding hybrids (shaded line) and the corresponding integrated DOS (thick red line) for CaN within the GGA approximation and its projection on the $\mathrm{Ca}$ and $\mathrm{N}$ atoms. We do not present the s-states which are deep in energy and contain one electron per spin-direction with their weight almost exclusively at the $\mathrm{N}$-sites. Notice that the $\mathrm{p}-\mathrm{d}$ hybrids can accommodate 3 electrons per spin direction. All three majority-spin states $(\sim 2.5$ at the $\mathrm{N}$ site and $\sim 0.5$ at the Ca site) are occupied, while only two out of three $(\sim 1.5$ at the $\mathrm{N}$ site and $\sim 0.5$ at the $\mathrm{Ca}$ site) are occupied resulting in a total spin magnetic moment of $1 \mu_{\mathrm{B}}$.

the values of the cationic integrated DOS at the Fermi level leads to small variation of the cationic spin magnetic moment around zero. At the $\mathrm{N}$ site we have about 2.5 states in the majority-spin band which are completely occupied while in the minority-spin bands we have about 2.35 states in total but only $\sim 1.5$ are occupied leading to a $\mathrm{N}$ spin magnetic moment of about $1 \mu_{B}$. Previous calculations led to a similar picture of the atom resolved spin-magnetic moments with the cation spin moments being one order of magnitude smaller than the anion spin magnetic moments ${ }^{7.30}$ Thus to conclude, we should have in all cases a total spin magnetic moment of $1 \mu_{B}$ since we have seven valence electrons, all compounds are halfmetals and the atom resolved spin moments fluctuate around 0 or $1 \mu_{B}$ for cations and anions, respectively, depending on the $\mathrm{p}-\mathrm{d}$ hybridization intensity.

\section{DEFORMATIONS}

To simulate deformation of the lattices with respect to equilibrium we took into account three cases: (i) hydro- 

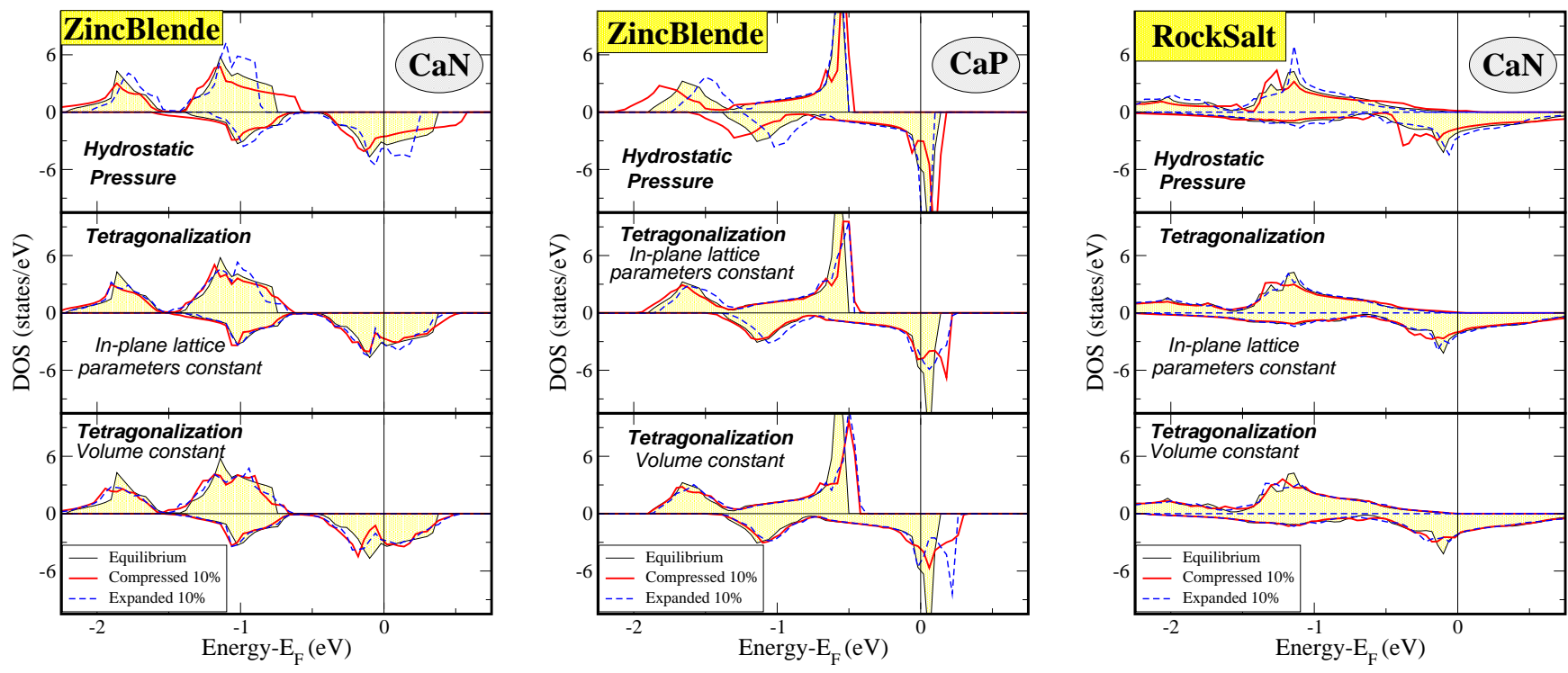

FIG. 6: (Color online) Total DOS of CaN (left panels) and CaP (middle panels) compounds in the zincblende structure and $\mathrm{CaN}$ (right panels) in the rocksalt structure within GGA at the equilibrium lattice constants compared to : (upper panel) $10 \%$ uniform expansion or contraction of the lattice as in the case of hydrostatic pressure, (middle panel) tetragonalization keeping the in-plane lattice parameters equal to the equilibrium ones and expanding or contracting the out-of-plane lattice parameter by $10 \%$, and (lower panel) tetragonalization expanding or contracting the in-plane lattice parameters by $10 \%$ and in the same time changing accordingly the out-of-plane lattice parameter to keep the unit cell volume constant to the equilibrium one.

TABLE II: Calculated spin magnetic moments (in $\mu_{B}$ ) for $\mathrm{CaN}$ and $\mathrm{CaP}$ in the zincblende structure and CaN in the rocksalt structure using the FPLO method within the GGA approximation. The first line corresponds to the ideal equilibrium lattice constants and we have considered three cases of deformation: (i) hydrostatic pressure (Hydro) which is a uniform compression and expansion of the cubic lattice and the percentage in the second column shows the change in the lattice constant ("-" means compression and "+" means expansion), (ii) tetragonalization of the lattice keeping the in-plane lattice parameters constant and equal to the equilibrium values and changing only the lattice parameter along the c-axis (Tetra-I) and the percentage in the second column shows this change, and (iii) tetragonalization where we vary the in-plane lattice parameters by the percentage shown in the second column and in the same time we vary also the lattice parameter along the c-axis so that the unit-cell volume is kept equal to the equilibrium one (Tetra-II). In all cases the total spin-magnetic is kept equal to $1.0 \mu_{B}$ (even in the case of $\pm 10 \%$ hydrostatic pressure where the unit-cell volume changes by almost $30 \%$ ) and thus the half-metallicity is preserved.

\begin{tabular}{|c|c|c|c|c|c|c|c|c|c|c|}
\hline & \multirow[b]{2}{*}{ Case } & \multicolumn{3}{|c|}{ CaN-ZincBlende $(5.45 \AA)$} & \multicolumn{3}{|c|}{ CaP-ZincBlende $(6.56 \AA)$} & \multicolumn{3}{|c|}{ 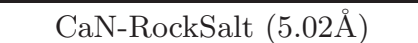 } \\
\hline & & $\mathrm{Ca}$ & $\mathrm{N}$ & Total & $\mathrm{Ca}$ & $\mathrm{P}$ & Total & $\mathrm{Ca}$ & $\mathrm{N}$ & Total \\
\hline Ideal & & -0.073 & 1.073 & 1.0 & 0.025 & 0.975 & 1.0 & -0.065 & 1.065 & 1.0 \\
\hline \multirow[t]{6}{*}{ Hydro } & $-1 \%$ & -0.072 & 1.072 & 1.0 & 0.025 & 0.975 & 1.0 & -0.061 & 1.061 & $\overline{1.0}$ \\
\hline & $-5 \%$ & -0.071 & 1.071 & 1.0 & 0.025 & 0.975 & 1.0 & -0.059 & 1.057 & 0.999 \\
\hline & $-10 \%$ & -0.068 & 1.068 & 1.0 & 0.026 & 0.974 & 1.0 & -0.048 & 1.017 & 0.969 \\
\hline & $+1 \%$ & -0.074 & 1.074 & 1.0 & 0.025 & 0.975 & 1.0 & -0.067 & 1.067 & 1.0 \\
\hline & $+5 \%$ & -0.075 & 1.075 & 1.0 & 0.026 & 0.974 & 1.0 & -0.070 & 1.070 & 1.0 \\
\hline & $+10 \%$ & -0.080 & 1.080 & 1.0 & 0.026 & 0.974 & 1.0 & -0.079 & 1.079 & 1.0 \\
\hline \multirow[t]{6}{*}{ Tetra-I } & $-1 \%$ & -0.072 & 1.072 & 1.0 & 0.025 & 0.975 & 1.0 & -0.063 & 1.063 & 1.0 \\
\hline & $-5 \%$ & -0.072 & 1.072 & 1.0 & 0.025 & 0.975 & 1.0 & -0.062 & 1.062 & 1.0 \\
\hline & $-10 \%$ & -0.071 & 1.071 & 1.0 & 0.026 & 0.974 & 1.0 & -0.058 & 1.055 & 0.997 \\
\hline & $+1 \%$ & -0.073 & 1.073 & 1.0 & 0.025 & 0.975 & 1.0 & -0.065 & 1.065 & 1.0 \\
\hline & $+5 \%$ & -0.073 & 1.073 & 1.0 & 0.026 & 0.974 & 1.0 & -0.066 & 1.066 & 1.0 \\
\hline & $+10 \%$ & -0.074 & 1.074 & 1.0 & 0.027 & 0.973 & 1.0 & -0.068 & 1.068 & 1.0 \\
\hline \multirow[t]{6}{*}{ Tetra-II } & $-1 \%$ & -0.073 & 1.073 & 1.0 & 0.026 & 0.974 & 1.0 & -0.064 & 1.064 & $\overline{1.0}$ \\
\hline & $-5 \%$ & -0.072 & 1.072 & 1.0 & 0.027 & 0.973 & 1.0 & -0.064 & 1.064 & 1.0 \\
\hline & $-10 \%$ & -0.071 & 1.071 & 1.0 & 0.027 & 0.973 & 1.0 & -0.063 & 1.063 & 1.0 \\
\hline & $+1 \%$ & -0.073 & 1.073 & 1.0 & 0.026 & 0.974 & 1.0 & -0.064 & 1.064 & 1.0 \\
\hline & $+5 \%$ & -0.072 & 1.072 & 1.0 & 0.027 & 0.973 & 1.0 & -0.064 & 1.064 & 1.0 \\
\hline & $+10 \%$ & -0.071 & 1.071 & 1.0 & 0.029 & 0.971 & 1.0 & -0.062 & 1.062 & 1.0 \\
\hline
\end{tabular}


static pressure (Hydro) which is a uniform compression and expansion of the cubic lattice, (ii) tetragonalization of the lattice keeping the in-plane lattice parameters constants and equal to the equilibrium values and changing only the lattice parameter along the c-axis (Tetra-I), and (iii) tetragonalization where we vary both the in-plane and out-of-plane parameters keeping the unit cell volume constant to the equilibrium (Tetra-II). In Table III we have gathered our results concerning the spin magnetic moments for $\mathrm{CaN}$ and $\mathrm{CaP}$ adopting the $\mathrm{ZB}$-lattice. We have also performed similar calculations for $\mathrm{SrN}$ and $\mathrm{SrP}$ but the behavior of the $\mathrm{Sr}$ compounds is identical to the Ca ones and thus we do not present them. Moreover we present results also for $\mathrm{CaN}$ in the rocksalt structure which has been also widely studied in literature. Preliminary results on $\mathrm{SrN}$ in both $\mathrm{ZB}$ and $\mathrm{RS}$ structure taking into account only the case of tetragonalization keeping the unit cell volume constant have been published in Ref. 31. The latter type of distortion is expected to occur more often when these compounds are grown on top of various substrates, but the other two cases under study are also susceptible to occur and we include them aiming to give a better description of the properties of these alloys under various distortions. In the second column of the table we present for each one of the three cases the percentage of change; "-" means compression by that percentage and "+" means expansion. Moreover in the case of the hydrostatic pressure the percentage refers to the lattice constant since the expansion or compression is uniform, in the case of Tetra-I where we keep constant the in-plane lattice parameters the percentage refers to the out-of-plane lattice parameter, and finally in case TetraII where we keep the unit-cell volume constant the percentage refers to the in-plane lattice constants. For each case we took six values of the percentage $\pm 1 \%, \pm 5 \%$ and $\pm 10 \%$. The larger changes in the unit cell volume occur for the Hydrostatic case where $\pm 10 \%$ change of the lattice constant means compression or expansion of the unit cell volume by about $27 \%$. Amazingly even for these extreme cases $\mathrm{CaN}$ and $\mathrm{CaP}$ in the $\mathrm{ZB}$ lattice remain half-metallic with a total spin magnetic moment of $1 \mu_{B}$. CaN in the RS-lattice shows similar behavior to the $\mathrm{ZB}$ one and only in the case of $10 \%$ compression it is at the verge of half-metallicity and Fermi level is located at the edge of the occupied majority-spin states; this behavior occurs because the half-metallic gap (energy difference between the upper edge of the occupied majority-spin states and the Fermi level) is $0.1 \mathrm{eV}$ much smaller than the compounds in the ZB lattice. Deformations in $\mathrm{CaN}$ lead to small changes of the absolute values of the $\mathrm{Ca}$ and $\mathrm{N}$ atomic spin magnetic moments of less than $0.07 \mu_{B}$ in such a way that they cancel each other keeping the total spin moment constant. When we replace $\mathrm{P}$ for $\mathrm{N}$ in $\mathrm{CaN}$ the fluctuations of the atomic spin magnetic moments of the constituent atoms are even smaller.

In Fig. 6 we present for the alloys in Table 1 the total DOS taking into account expansion or compression by $10 \%$ which are the limiting cases studied and compare them with the DOS for the ideal cubic lattices at the equilibrium lattice constants. We focus on the energy window including the p-d bonding hybrids which are the most relevant for magnetism. In the case of the $\pm 1 \%$ and $\pm 5 \%$ cases the DOS for the distorted lattices coincide with the DOS for the equilibrium lattice and the curves fall almost one on top of the other; the only noticeable difference concerns the hydrostatic pressure case where compression leads to slightly wider bands and thus smaller pick intensities. Differences in the DOS are only visible in the case of the $\pm 10 \%$ deformations presented in the figure. The most important changes occur in the case of hydrostatic pressure where compression leads to wider bands and expansion to narrower bands, this behavior being typical for p-orbitals. In the case of tetragonalization the ZB-CaN shows very small changes in the DOS shape while for ZB-CaP we remark a small redistribution of the weight of the empty minority-spin states just above the Fermi level. For CaN in the RS-lattice the changes in the DOS are even more marginal than for the ZB-lattice. The large half-metallic gaps especially in the case of the ZB lattice ensure that any small change in the characteristics of the DOS does not affect the half-metallicity and the Fermi level still crosses exclusively the minority-spin bands. The same image is also valid for the $\mathrm{SrN}$ and $\mathrm{SrP}$ alloys. Thus we can safely conclude that deformation of the lattice only marginally affects the electronic properties as was the case also for the magnetic properties discussed just above.

\section{CONCLUSIONS}

We have studied the properties of the $\mathrm{XY}(\mathrm{X}=\mathrm{Mg}$, $\mathrm{Ca}$, Sr and $\mathrm{Y}=\mathrm{N}, \mathrm{P}, \mathrm{As}, \mathrm{Sb}$ ) pnictides crystallizing in the zincblende structure using a full-potential electronic structure method in conjunction to the generalized gradient approximation. We have shown that the $\mathrm{Ca}$ - and Sr-based alloys should be classified as sp-electron ferromagnets (also known as $d^{0}$-ferromagnets). Moreover they are half-metals since the Fermi level crosses only minority-spin states and the total spin magnetic moment per formula unit was found to be $1.0 \mu_{B}$. Contrary to the $\mathrm{Ca}$ and $\mathrm{Sr}$ alloys, in the case of the $\mathrm{Mg}$ alloys the $\mathrm{p}-\mathrm{d}$ hybridization effect is much weaker and only $\mathrm{MgN}$ is a half-metallic ferromagnet while $\mathrm{MgP}, \mathrm{MgAs}$ and $\mathrm{MgSb}$ prefer energetically to be non-magnetic. We have also studied for these alloys the effect of deformation taking into account both the cases of hydrostatic pressure and tetragonalization keeping constant either the in-plane lattice parameters or the unit cell volume. Even large degrees of deformation only marginally affected the electronic and magnetic properties of these alloys which kept their half-metallic ferromagnetic character. Similar behavior is found also when they crystallize in the rocksalt instead of the zincblende structure. Our results suggest that the alkaline-earth metal monopnictides are promis- 
ing materials for spintronic applications.

* Electronic address: kozdogan@yildiz.edu.tr

† Electronic address: galanakis@upatras.gr

1 I. Žutić, J. Fabian, and S. Das Sarma, Rev. Mod. Phys. 76, 323 (2004).

2 C. Felser, G. H. Fecher, and B. Balke, Angew. Chem. Int. Ed. 46, 668 (2007).

${ }^{3}$ H. Zabel, Materials Today 9, 42 (2006).

4 M. I. Katsnelson, V. Yu. Irkhin, L. Chioncel, A. I. Lichtenstein, and R. A. de Groot, Rev. Mod. Phys. 80, 315 (2008).

5 O. Volnianska and P. Boguslawski, J. Phys.:Condens. Matter 22, 073202 (2010).

6 Ph. Mavropoulos and I. Galanakis, J. Phys. Condens. Matter 19, 315221 (2007).

7 M. Geshi, K. Kusakabe, H. Tsukamoto, and N. Suzuki, 2004 Preprint arXiv:cond-mat/0402641 (2004).

8 K. Kusakabe, M. Geshi, H. Tsukamoto, and N. Suzuki, J. Phys.:Condens. Matter 16, S5639 (2004).

9 J. Chen, G. Y. Gao, K. L. Yao, and M. H. Song, J. All. Cmps. 509, 10172 (2011).

10 X. Liu, B. Lu, T. Iimori, K. Nakatsuji, and F. Komori, Surf. Sci. 602, 1844 (2008).

11 M. Sieberer, J. Redinger, S. Khmelevskyi, and P. Mohn, Phys. Rev. B 73, 024404 (2006).

12 C.-W. Zhang, J. Phys. D.: Appl. Phys. 41, 085006 (2008).

13 K. Zberecki, L. Adamowicz, and M. Wierzbicki, Phys. St. Solidi (b) 246, 2270 (2009).

14 E. Yan, Physica B 407, 879 (2012).

15 G. Y. Gao, K. L. Yao, Z. L. Liu, Y. Min, J. Zhang, S. W. Fan, and D. H. Zhang, J. Phys.:Condens. Matter 21, 275502 (2009).

16 G. Y. Gao, K. L. Yao, M. H. Song, and Z. L. Liu, J. Magn. Magn. Mater. 323, 2652 (2011).

17 G. Y. Gao, K. L. Yao, Z. L. Liu, J. L. Jiang, L. H. Yu, and Y. L. Shi, J. Phys.:Condens. Matter 19, 315222 (2007).
18 G. Y. Gao, K. L. Yao, E. Şaşıŏlu, L. M. Sandratskii, Z. L. Liu, and J. L. Jiang, Phys. Rev. B 75, 174442 (2007).

19 G. Y. Gao and K. L. Yao, Appl. Phys. Lett. 91, 082512 (2007).

20 S. Dong and H. Zhao, Appl. Phys. Lett. 98, 182501 (2011).

21 C.-W. Zhang, S.-S Yan, and H. Li, Phys. St. Solidi (b) 245, 201 (2008).

22 G. Y. Gao and K.-L. Yao, J. Appl. Phys. 106, 053703 (2009).

23 U. P. Verma, Mohini, P. S. Bisht, and P. Jensen, Semicond. Sci. Technol. 25, 105002 (2010).

24 K. L. Yao, J. L. Jiang, Z. L. Liu, and G. Y. Gao, Phys. Lett. A 359, 326 (2006).

${ }^{25}$ Y. Li and J. Yu, Phys. Rev. B 78, 165203 (2008).

${ }^{26}$ O. Volnianska and P. Boguslawski, Phys. Rev. B 75, 224418 (2007).

27 M. Geshi, K. Kusakabe, H. Nagara, and N. Suzuki, Phys. Rev. B 76, 054433 (2007).

${ }^{28}$ G. Y. Gao, K. L. Yao, Z. L. Liu, J. Zhang, Y. Min, and S. W. Fan, Phys. Lett. A 372, 1512 (2008).

29 A. Droghetti, N. Baadji, and S. Sanvito, Phys. Rev. B 80, 235310 (2009).

30 A. Laref, E. Şaşığlu, and I. Galanakis, J. Phys.:Condens. Matter 23, 296001 (2011).

31 K. Özdoğan, E. Şaşıŏ̆lu, and I. Galanakis, submitted; preprint arXiv:1203.1106 (2012).

32 G. Y. Gao, K. L. Yao, and N. Li, J. Phys.:Condens. Matter 23, 075501 (2011).

33 K. Koepernik and H. Eschrig, Phys. Rev. B 59, 1743 (1999).

34 J. P. Perdew and Y. Wang, Phys. Rev. B 45, 13244 (1992).

35 J. P. Perdew, K. Burke, and M. Ernzerhof, Phys. Rev. Lett. 78, 1396 (1997). 